\title{
Cortical Coordination Dynamics and the Disorganization Syndrome in Schizophrenia
}

\author{
Steven L Bressler*, \\ 'Center for Complex Systems \& Brain Sciences, Florida Atlantic University, Boca Raton, FL, USA
}

\begin{abstract}
There has been a long history of investigation in the fields of neuropsychology and cognitive psychology into the question of functional integration in the brain. Each of the several dominant themes in that history can be interpreted as representing an important feature of a unitary general mechanism that integrates distributed processes in the cerebral cortex. This mechanism must allow local areas to function within the large-scale anatomical structure of the cortex so as to satisfy competing requirements for stability and flexibility. Each specialized cortical area must perform a unique role by expressing its own form of information, yet must have its performance constrained by interactions with other areas to which it is connected. In order to generate adaptive behavior within changing and not fully predictable environments, the cortex as a whole must be able to rapidly coordinate the activities of variable assemblages of areas that can collectively express consensual information that is appropriate for the functional requirements engendered by each successive stage of behavioral performance. This paper proposes that the phase synchronization of neuronal population activity from different cortical areas may serve a role in large-scale coordination. Theoretical studies suggest that the cortex normally operates in a metastable dynamic regime in which groups of areas are able to coordinate rapidly and reversibly their activities through changes in their degree of phase synchronization. A disruption of phase synchronization, leading to an excess of local information expression by cortical areas, is proposed as a contributing factor to the disorganization syndrome in schizophrenia.

Neuropsychopharmacology (2003) 28, S35-S39. doi: I0.1038/sj.npp. 1300145
\end{abstract}

Keywords: cerebral cortex; coordination; disorganization; dynamics; phase synchronization; schizophrenia

\section{INTRODUCTION}

Normal cognition requires the precise spatial and temporal organization of numerous brain processes. Cognitive microstates ordinarily follow one another in a progression that is meaningfully consistent with the physical and social environments. The makeup of these states and their ordering depend on the integration of a variety of different functions in a distributed collection of brain regions, and the orderly flow of thought processes may be disturbed when mechanisms responsible for that integration fail. Understanding the mechanisms by which the functions of different brain structures are integrated in a coherent manner is essential for a neural account of cognition and its disruption in disease processes.

The first aim of this article is to consider the question of functional integration in the brain. The functional attributes of neurons in specific brain structures are clearly important, as are the local circuit properties in those structures.

* Correspondence: Dr SL Bressler, Center for Complex Systems \& Brain Sciences, Florida Atlantic University, 777 Glades Road, Boca Raton, FL 3343I, USA, Tel: + I 561297 2322, Fax: + 5612973634 , E-mail: bressler@fau.edu

Received 0I September 2002; revised 03 November 2002; accepted 03 December 2002
Equally relevant are the large-scale connectional architecture that join these structures together in global systems. As important as these factors are, they are not sufficient for understanding functional integration in the brain. What is needed is a mechanism for the integration of local circuits within the large-scale anatomical structure. The second goal here is to discuss a mechanism that has been proposed for the cerebral cortex to explain how potentially conflicting neurocognitive processes may be quickly and efficiently reconciled (Bressler and Kelso, 2001; Bressler, 2002). A key feature of the proposed mechanism is that it is based on the ability of multiple cortical areas to rapidly and reversibly coordinate their population activities. Finally, the third goal is to advance the hypothesis that disruption of this coordination mechanism is a contributing factor in the disorganization syndrome, a psychopathological dimension in schizophrenia (Cuesta and Peralta, 2001).

\section{HISTORICAL PERSPECTIVE ON FUNCTIONAL INTEGRATION IN THE BRAIN}

The question of functional integration has long played an important role in the history of psychology, being central to a number of critical issues. Here, I briefly consider two issues regarding the nature of functional integration in the 
mind and brain. Each issue has been debated over long periods of time, and the present purpose is not to argue for one side or another, nor even to enter into a discussion of the relative merits of the arguments. Over long decades of discussion, neither of these debates has been resolved. However, the lack of resolution in a debate does not necessarily mean that the arguments on both sides are without merit. Rather, it may be taken as evidence that both views reflect valid, albeit partial, aspects of a multifaceted issue. Each of these two debates will be described only cursorily for the purpose of identifying the opposing viewpoints that are involved. It will be claimed that these views represent partial perspectives that can be used to identify complementary properties of a single brain process. The goal will be to consider what kind of mechanism could naturally account for all of the identified properties.

The first controversy to be considered concerns the classical antagonism between associationism and structuralism, which represents a major theme in the history of cognitive psychology. The concept of cognition as association was developed by the British philosophical psychologists of the 18th and 19th centuries: Locke, Hartley, Hume, James Mill, and his son, John Stuart Mill. The tradition reached full expression in the work of Bain, who devised a systematic psychology of association (Young, 1990). An important implication of associationism is that cognitive organization is built up by association based on statistical regularities existing in sensory data. Associationism continued unabated into the 20th century as a central tenet in the behaviorism of Pavlov, Watson, and Skinner. Then, in the second half of the 20th century, associationism came under assault by the psycholinguistic school of Chomsky, who shifted the emphasis in cognitive psychology to consideration of structure-sensitive processing. According to Chomskian structuralism, associationism merely addresses the surface appearance of cognitive relations without considering the deep structure underlying those relations. Cognitive organization is viewed as a syntactically and semantically ordered structure having law-like regularities. In contrast to associationism, these regularities are considered to be innate in the brain rather than acquired through experience (Deese, 1971). Both associationism and structuralism continue as competing themes in cognitive psychology today.

The second controversy, this time in the realm of neuropsychology, concerns the way in which brain function is allocated. Again, we find a debate characterized by two extreme views: localizationism and globalism, both of which have long histories (Zola-Morgan, 1995). Localizationism is the view that cognitive functions are localized to specific brain centers, cortical areas in particular. It can be traced back, in the modern era, to the phrenology of Gall and Spurzheim in the late 18th and early 19th centuries. The localizationist doctrine gradually took shape over subsequent decades through the work of numerous investigators, prominent among them being Broca, Fritsch and Hitzig, Munk, Ferrier, and Penfield, and continues today. The opposing view, which we may call globalism, refers to any theory holding that cognitive functions are globally distributed over the entire cortex. The origin of this perspective is often attributed to the work of Flourens, who in the early 19th century took a holistic approach to brain function in his criticism of phrenological thought (Deacon, 1989). Globalism also flourished during the ensuing years, notably through the contributions of Jackson, Goltz, Goldstein, Head, and Lashley, and has also persisted until the present time.

\section{THE DYNAMICAL SYSTEMS APPROACH TO FUNCTIONAL INTEGRATION}

To approach a resolution of these issues, we may turn to another, lesser known, tradition in neuropsychology, which holds that complex functions are implemented dynamically by distributed sets of neural structures briefly linked together within the connectional structure of the brain. The roots of this outlook can be traced to Wernicke, who stated that 'primary functions alone can be referred to specific cortical areas... all processes which exceed these primary functions... are dependent upon the fiber bundles connecting different areas of the cortex' (Eggert, 1977). This concept was further developed and refined by Pavlov, who envisioned complex neural function as resulting from the dynamic aggregation of distributed brain centers temporarily linked together in a common task. In the Pavlovian tradition, complex function is the property of a 'functional system' that is distributed and variable in its composition, and adaptive in its operation (Luria, 1962). The view of functional integration in the brain as the product of dynamic distributed systems has continued in modern times. For example, Mountcastle (1998) describes higher neural function in terms of interacting ensembles having 'dynamic properties not predictable from those of [the] individual members'.

In the light of the dynamical systems perspective, the opposing sides of the two controversies presented in the previous section may be understood as providing unique perspectives on neurocognitive function that are not necessarily mutually exclusive, and may actually be complementary. In fact, when taken together within the context of the modern understanding of cerebral cortical function, they may be seen to represent general, mutually supportive neurocognitive properties. If one considers the large number of identified cortical areas and their high degree of interconnectivity (Felleman and Van Essen, 1991), it is reasonable to assume that some mechanism must exist to associate the population activity in different cortical areas, and that this mechanism will be subject to an enormous degree of structural constraint because of the patterning of interareal anatomical connections. Furthermore, since cortical areas are known to have unique elementary functions (Posner et al, 1988), large-scale ${ }^{1}$ function could arise naturally through the association of population activity in multiply connected areas.

What is needed to elucidate this position is a reinterpretation of the concept of 'association' in the brain from the dynamical systems point of view. Many dynamical systems in biology are composed of a large number of spatially

\footnotetext{
${ }^{1}$ A critical distinction exists between the terms 'large scale' and 'global' in regard to functional integration. Large-scale function refers to the joint operation of neural populations that are distributed but localized, possibly including only a small fraction of all potential populations. Global function, by contrast, implies the involvement of all populations.
} 
distributed, interconnected components that have the potential of interacting. A general property of such systems is that the system components interact recurrently in a complex manner, leading to the emergence of observable system-wide patterns of interrelationship. This property can be usefully described in terms of the system's coordination dynamics (Kelso, 1994). 'Coordination' refers to the tendency of system parts to be functionally related, and 'coordination dynamics' refers to the way in which their relations change with time. Thus, the coordination of components in a dynamical system is a way of describing their functional association.

Coordination phenomena are ubiquitous in both motor and perceptual behavior. Coordination is a hallmark of the motor system, and is obvious in such motor behaviors as locomotion, bimanual manipulation, and unimanual manipulation. Speech is another common motor behavior requiring the precise coordination of multiple system components (muscle groups). Although not as often appreciated, coordination is just as prevalent in the perceptual systems as in the motor system. For example, coordination is required between the sensory modalities, such as when auditory and visual features must be related to recognize an object. It is also necessary for the spatial and temporal registration of the multiple features of objects within sensory modalities. Coordination must be at play in a wide variety of cognitive functions as well. Working memory, for instance, requires the precise coordination of storage and retrieval processes with perceptual, planning, and decision-making functions.

The widespread need for coordination in perceptual and motor behavior suggests that a common brain mechanism may exist to coordinate distributed neural processes that must cooperate in a joint function. Such a mechanism would be expected to meet certain criteria. First, to operate over entire systems it should coordinate the activity of neuronal ensembles rather than just single neurons. Second, in the cortex, these ensembles should be coordinated both locally within individual cortical areas and on a large scale across distributed areas. Third, it should allow for spontaneous variation in the degree of coordination as the cortical system dynamics evolve over time. A candidate mechanism that satisfies these conditions is the re-entrant interaction that continually occurs within and between cortical areas (Tononi et al, 1992).

From a dynamical systems viewpoint, understanding the effects of such re-entrant interactions on the cortical system may come from a description in terms of coupled oscillators (Bressler and Kelso, 2001). The portrayal of the cortical ensemble as an oscillator derives simply from the observation that ensemble activity continuously varies in time within bounds around a central value. The study of coupled oscillator systems has provided mathematical models that are able to account for coordinative interactions in a variety of systems. A critical system variable that is found in many such complex interacting systems is the relative phase between component oscillators. From the experimental observation of cortical local field potentials, reflecting ensemble activity, in monkeys performing visuomotor tasks, the relative phase is seen to take on all possible values over time, but at particular times to become concentrated around particular central values (Bressler and Kelso, 2001). Thus, the degree of relative phase concentration between different ensembles in the cortical system, reflecting their degree of coordination, varies over time and may change rapidly with changes in cognitive state.

Coupled oscillator systems often display metastability, the tendency for attraction to preferred relative phase relations without the existence of actual attracting fixed points, which would capture the system's coordination dynamics at a particular phase relation and prevent it from visiting other phase relations (Bressler and Kelso, 2001). In the metastable regime of its coordination dynamics, the system operates in a state that allows both integration and segregation of function: individual oscillators are balanced in their tendency for independent activity and their tendency to be coupled with the entire system. Thus, by providing a dynamic balance between independence and interaction, metastability could allow rapid changes to occur in the coordination dynamics of distributed cortical areas without them normally becoming locked in fixed phase relations.

Based on these considerations, we can envision the cortical system as a whole having the ability to rapidly coordinate particular subsets of component ensembles through increased relative phase concentration as needed for cognitive performance. The ability of particular ensembles to become coordinated by phase synchronization must, of course, depend heavily on the anatomical constraints imposed on interareal re-entrant interactions by the architecture of cortical connectivity, so that any two ensembles in the cortex have the potential to become coordinated by phase synchronization if axonal pathways exist to allow their re-entrant interaction. In this perspective, then, phase synchronization of ensemble activity is a basic mechanism of cortical association. The large-scale patterning of phase-synchronized ensembles is structured by the anatomical patterning of cortical interareal connectivity, and its temporal evolution may be subject to structure-sensitive rules regarding the recruitment and exclusion of areas. Through the property of metastability, this mechanism may allow expression of local cortical area dynamics as well as global dynamics at the level of the entire cortex. The way in which local information may be expressed and combined globally is considered next.

Since cortical areas have unique topological positions in the overall connectional structure of the cortex, each area is typically believed to process a different form of information. Following Jackendoff (1996), the 'form of information' is taken to be 'an organized combinatorial space of distinctions available to the brain'. We may consider this informational state space (Churchland and Sejnowski, 1994) to be created within each area's specialized local associative memory (Pulvermuller, 1999), and the information to be expressed by the creation of local spatial activity patterns (Ohl et al, 2001). According to this view, the generation of a spatial activity pattern by a local cortical area represents the selection of one state from the space of all possible states in which the activity could be (Jackendoff, 1996). Through re-entrant interactions between connected cortical areas, mutual constraints are imposed on the generation of spatial activity patterns in those areas, thus restricting the regions of their state spaces that can be accessed by their activities. The imposition of constraints 
on one area from interactions with the other areas to which it is connected creates a context for that area's local processing. Local context may have a number of important consequences, such as dynamic grouping in vision (Watt and Phillips, 2000).

With large numbers of cortical areas undergoing reentrant interactions during cognitive processing, some influences are expected to be in conflict, while others should be mutually consistent. In order to produce a unified and coherent cognitive state, a set of coordinated cortical areas must emerge to express informationally consistent local spatial activity patterns. Owing to metastability, a different set of coordinated areas can emerge rapidly and smoothly at each stage of cognitive processing, a set that mutually satisfies the multiple constraints in effect at that stage. The manifestation of consensual local spatial activity patterns in coordinated cortical areas represents a process of immense combinatorial power, encompassing not only all the possible combinations of coordinated areas, but also all the possible combinations of states of those coordinated areas.

The idea that satisfaction of multiple constraints can produce a unified consensual state is also found in the theory of cognitive coherence (Thagard, 2000). Humans have an inherent facility for constructing inferences about the world based on sensory inputs. According to this theory, the coherence of an inference comes from the satisfaction of multiple constraints according to coherence and incoherence relations, and mathematically precise procedures have been devised to maximize measures of coherence. This idea of cognitive coherence based on multiple constraint satisfaction fits naturally with the model presented here of neural coherence resulting from consensual spatial activity patterns in coordinated cortical areas.

\section{THE DISORGANIZATION SYNDROME AS A DISTURBANCE OF CORTICAL COORDINATION DYNAMICS}

This final section considers a potential consequence of disruption in the phase synchronization mechanism that was proposed in the previous section to account for the creation of consensual spatial activity patterns in distributed cortical areas with the resultant emergence of coherent cognitive states. Such disruption is suggested as a contributing factor to the disorganization syndrome in schizophrenia, which has long been deemed to be a condition of impaired cognitive association (Haig et al, 2000). As discussed above, association is interpreted in the dynamical systems description as phase synchronization between cortical areas. From this perspective, then, disorganization is viewed as a disorder of the metastable balance between large-scale integration and independent processing in the cortex, in favor of independent processing. One predicted consequence would be that large-scale patterns of neural population coordination, were they to be measured in schizophrenic patients, would be diminished and disjointed, as compared to normals.

Disordered coordination of this type would also be expected to produce a lack of mutual constraint between connected cortical areas, leading to the excessive expression of local information unrestrained by large-scale context.
Connected areas that would normally produce consensual spatial activity patterns would tend to express information that was in conflict. Unrestrained local cortical information expression could take many forms, but would be predicted to produce experience and behavior that are lacking coherence and appearing out of context (Silverstein and Schenkel, 1997; Cohen et al, 1999). One particular manifestation of this might be the misperception by schizophrenic patients of intrinsically generated sensory activity as being extrinsically generated. Frith and Dolan (1996) suggest that such misperception results from the disassociation of prefrontal cortex from those posterior cortical areas that generate the sensory activity.

One factor that has been considered as a cause of disorganization in schizophrenia is a deficiency of anatomical connectivity between cortical areas (Hoffman and McGlashan, 1998). Neuroimaging studies (Josin and Liddle, 2001) and visual psychophysics studies (Silverstein et al, 2000; Izawa and Yamamoto, 2002) provide evidence for the reduced functional connectivity in schizophrenia that would be expected to result from such anatomical disconnection if the ability of cortical areas to become phase synchronized were impaired. Silverstein et al (2000) suggest that the deterioration of perceptual organization observed in schizophrenia is one manifestation of a broader deficit in contextual coordination operating across cognitive domains, noting that schizophrenia is marked by a contextual breakdown in linguistic and attention functions, as well as vision. Such a broad range of contextual impairments is exactly the type of general consequence to be expected from the failure of a common interareal coordination mechanism. It is to be hoped that a deeper neurophysiological and neuropharmacological understanding of this mechanism could lead to improved diagnostic and therapeutic procedures for the treatment of schizophrenia.

\section{REFERENCES}

Bressler SL (2002). Understanding cognition through large-scale cortical networks. Curr Dir Psychol Sci 11: 58-61.

Bressler SL, Kelso JA (2001). Cortical coordination dynamics and cognition. Trends Cognit Sci 5: 26-36.

Churchland PS, Sejnowski TJ (1994). The Computational Brain. MIT Press: Cambridge, MA.

Cohen JD, Barch DM, Carter C, Servan-Schreiber D (1999). Context-processing deficits in schizophrenia: converging evidence from three theoretically motivated cognitive tasks. J Abnorm Psychol 108: 120-133.

Cuesta MJ, Peralta V (2001). Integrating psychopathological dimensions in functional psychoses: a hierarchical approach. Schizophr Res 52: 215-229.

Deacon T (1989). Holism and associationism in neuropsychology: an anatomical synthesis. In: Perecman E (ed). Integrating Theory and Practice in Clinical Neuropsychology. Erlbaum: Hillsdale. pp 1-47.

Deese J (1971). Psycholinguistics. Allyn \& Bacon: Boston.

Eggert GH (1977). Wernicke's Works on Aphasia: A Sourcebook and Review. Mouton Publishers: The Hauge.

Felleman DJ, Van Essen DC (1991). Distributed hierarchical processing in the primate cerebral cortex. Cereb Cortex 1: 1-47. Frith C, Dolan R (1996). The role of the prefrontal cortex in higher cognitive functions. Cogn Brain Res 5: 175-181. 
Haig AR, Gordon E, De Pascalis V, Meares RA, Bahramali H, Harris A (2000). Gamma activity in schizophrenia: evidence of impaired network binding? Clin Neurophysiol 111: 1461-1468.

Hoffman RE, McGlashan TH (1998). Reduced corticocortical connectivity can induce speech perception pathology and hallucinated 'voices'. Schizophr Res 30: 137-141.

Izawa R, Yamamoto S (2002). Spatio-temporal disintegration of visual perception in schizophrenia as revealed by a novel cognitive task, the Searchlight Test. Schizophr Res 53: 67-74.

Jackendoff R (1996). Languages of the Mind. MIT Press: Cambridge, MA.

Josin GM, Liddle PF (2001). Neural network analysis of the pattern of functional connectivity between cerebral areas in schizophrenia. Biol Cybernet 84: 117-122.

Kelso JAS (1994). Elementary coordination dynamics. In: Swinnen SP, Heuer H, Massion J, Casaer P (eds). Interlimb Coordination: Neural, Dynamical, and Cognitive Constraints. Academic Press: New York. pp 301-318.

Luria AR (1962). Higher Cortical Functions in Man. Basic Books: New York.

Mountcastle V (1998). Perceptual Neuroscience: The Cerebral Cortex. Harvard University Press: Cambridge, MA.

Ohl FW, Scheich H, Freeman WJ (2001). Change in pattern of ongoing cortical activity with auditory category learning. Nature 412: 733-736.
Posner MI, Petersen SE, Fox PT, Raichle ME (1988). Localization of cognitive operations in the human brain. Science 240: 1627-1631.

Pulvermuller F (1999). Words in the brain's language. Behav Brain Sci 22: 253-336.

Silverstein SM, Kovacs I, Corry R, Valone C (2000). Perceptual organization, the disorganization syndrome, and context processing in chronic schizophrenia. Schizophr Res 43: 11-20.

Silverstein SM, Schenkel LS (1997). Schizophrenia as a model of context-deficient cortical computation. Behav Brain Sci 20: 696697.

Thagard P (2000). Coherence in Thought and Action. MIT Press: Cambridge, MA.

Tononi G, Sporns O, Edelman GM (1992). Reentry and the problem of integrating multiple cortical areas: simulation of dynamic integration in the visual system. Cereb Cortex 2: 310335.

Watt RJ, Phillips WA (2000). The function of dynamic grouping in vision. Trends Cogn Sci 4: 447-454.

Young RM (1990). Mind, Brain and Adaptation in the Nineteenth Century: Cerebral Localization and its Biological Context from Gall to Ferrier. Oxford University Press: New York.

Zola-Morgan S (1995). Localization of brain function: the legacy of Franz Joseph Gall (1758-1828). Annu Rev Neurosci 18: 359-383. 